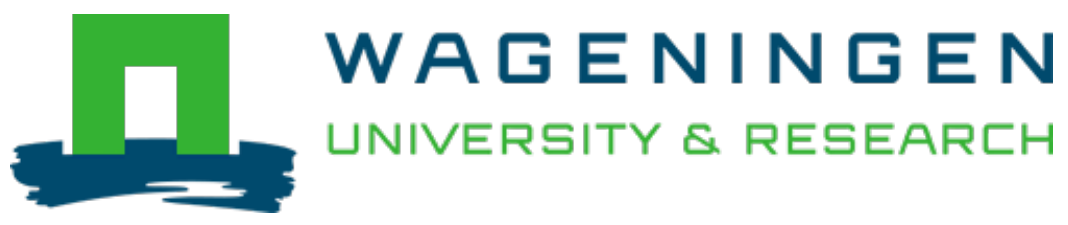

\title{
A Resurrected Scenario : Single Gain and Massive Loss of Nitrogen-Fixing Nodulation
}

\author{
Trends in Plant Science \\ Velzen, Robin; Doyle, Jeff J.; Geurts, Rene \\ https://doi.org/10.1016/j.tplants.2018.10.005
}

This article is made publicly available in the institutional repository of Wageningen University and Research, under the terms of article $25 \mathrm{fa}$ of the Dutch Copyright Act, also known as the Amendment Taverne. This has been done with explicit consent by the author.

Article 25 fa states that the author of a short scientific work funded either wholly or partially by Dutch public funds is entitled to make that work publicly available for no consideration following a reasonable period of time after the work was first published, provided that clear reference is made to the source of the first publication of the work.

This publication is distributed under The Association of Universities in the Netherlands (VSNU) 'Article $25 \mathrm{fa}$ implementation' project. In this project research outputs of researchers employed by Dutch Universities that comply with the legal requirements of Article $25 \mathrm{fa}$ of the Dutch Copyright Act are distributed online and free of cost or other barriers in institutional repositories. Research outputs are distributed six months after their first online publication in the original published version and with proper attribution to the source of the original publication.

You are permitted to download and use the publication for personal purposes. All rights remain with the author(s) and / or copyright owner(s) of this work. Any use of the publication or parts of it other than authorised under article $25 \mathrm{fa}$ of the Dutch Copyright act is prohibited. Wageningen University \& Research and the author(s) of this publication shall not be held responsible or liable for any damages resulting from your (re)use of this publication.

For questions regarding the public availability of this article please contact openscience.library@wur.nl 


\title{
A Resurrected Scenario: Single Gain and Massive Loss of Nitrogen-Fixing Nodulation
}

\author{
Robin van Velzen, ${ }^{1}$ Jeff J. Doyle, ${ }^{2}$ and Rene Geurts $\mathbb{B}^{1, *}$
}

Root nodule endosymbiosis with nitrogen-fixing bacteria provides plants with unlimited access to fixed nitrogen, but at a significant energetic cost. Nodulation is generally considered to have originated in parallel in different lineages, but this hypothesis downplays the genetic complexity of nodulation and requires independent recruitment of many common features across lineages. Recent phylogenomic studies revealed that genes that function in establishing or maintaining nitrogen-fixing nodules are independently lost in non-nodulating relatives of nitrogen-fixing plants. In our opinion, these data are best explained by a scenario of a single gain followed by massively parallel loss of nitrogenfixing root nodules triggered by events at geological scale.

\section{Nitrogen-Fixing Nodule Endosymbiosis: A Complex Genetic Trait}

Nitrogen is a critical limiting element for plant growth. It is predominantly present as atmospheric di-nitrogen gas $\left(\mathrm{N}_{2}\right)$, an unsuitable source for plants. Instead, plants rely on reduced nitrogen forms, such as ammonium $\left(\mathrm{NH}_{4}^{+}\right)$or nitrate $\left(\mathrm{NO}_{3}{ }^{-}\right)$that they generally absorb from soil. Some plants, however, make specialized root organs called nodules where they intracellularly host diazotrophic (see Glossary) bacteria. Legumes (Fabales, Fabaceae) and the nonlegume Parasponia (Rosales, Cannabaceae) host a polyphyletic group of diazotrophic $\alpha$ - and $\beta$-proteobacteria collectively referred to as rhizobia. Other nodulating species are known as 'actinorhizal' because they host diazotrophic filamentous Actinobacteria in the genus Frankia. Inside nodule cells, these microsymbionts find appropriate physiological conditions to catalyze the conversion of atmospheric $\mathrm{N}_{2}$ to $\mathrm{NH}_{4}{ }^{+}$by the bacterial enzyme complex nitrogenase [1]. Fueled by plant photosynthates, such endosymbionts provide these plants with an additional supply of nitrogen.

Nitrogen fixation is an energy-demanding conversion, requiring 16 moles of ATP per mole of $\mathrm{N}_{2}$ fixed [1]. Nodulating plants tightly regulate nodule numbers through autoregulation [2,3] and abolish nodulation altogether when sufficient nitrogen is available, such as in fertilized agricultural fields [4]. Similarly, nodulation has been found to be limited mainly by photosynthesis in tropical legumes [5]. Thus, it is clear that nodulation confers a fitness advantage only under environmental conditions in which growth is limited by nitrogen and when the benefit conferred by symbiotic nitrogen exceeds the cost of photosynthetic carbon.

Nitrogen-fixing nodules are the result of an intricate exchange of host plant and microsymbiont signals that simultaneously trigger nodule organogenesis and intracellular microbial infection. Within nodule cells, specialized symbiotic membranes allow for the exchange of sugars and ammonia, while defense responses are repressed $[6,7]$. Genetic dissection of nodulation in the legume models Medicago truncatula (medicago) and Lotus japonicus (lotus) uncovered more than 40 essential symbiosis genes. These include genes encoding LysM-type receptor kinases such as NFP/NFR5 that perceive rhizobial lipo-chitooligosaccharides (LCOs; known as Nod

\section{Highlights}

$\mathrm{N}_{2}$-fixing nodulation symbiosis is a complex and important agronomic trait. It occurs in phylogenetically separated lineages, and its evolution may be explained by two alternative hypotheses: (i) single gain followed by massively parallel loss, or (ii) parallel evolution and fewer losses. The latter hypothesis is widely accepted, but the first hypothesis is supported by recent phylogenomic data.

Molecular and developmental commonalities across distinct lineages support a common origin of nodulation. Moreover, recent comparative genomics studies revealed parallel loss of key nodulation genes in non-nodulating species.

These findings support a single gain of nodulation followed by massively parallel loss in most descendant lineages. Such massive loss may have been triggered by reductions in global atmospheric $\mathrm{CO}_{2}$ levels.

${ }^{1}$ Laboratory of Molecular Biology, Department of Plant Sciences, Wageningen University \& Research, 6708PB, Wageningen, The Netherlands

${ }^{2}$ School of Integrative Plant Science, Section of Plant Breeding \& Genetics and Section of Plant Biology, 240 Emerson Hall, Cornell University, Ithaca, NY 14853, USA

${ }^{*}$ Correspondence: rene.geurts@wur.nl (R. Geurts). 


\section{Trends in Plant Science}

\section{CellPress}

REVIEWS

factors) and activate a transcriptional network initiating nodule organogenesis, of which NIN is a master regulator $[6,8,9]$. Together with a series of other genes, such as $\boldsymbol{R P G}, N I N$ is also essential for intracellular infection $[8,10]$. Considering the developmental and physiological complexity and the number of genes that are indispensable for nodule formation, it is clear that nitrogen-fixing nodulation symbiosis is a functionally and genetically complex trait.

Recent comparative genomic studies have provided insights into the evolution of the nodulation trait $[11,12]$. Here, we discuss the implications of these new findings and advocate a radical change in the current view on evolution of nitrogen-fixing nodulation.

\section{Phylogenetic Perspective on Nitrogen-Fixing Root Nodules}

Because all nodulating plant species occur in the monophyletic group comprising the orders Fabales, Fagales, Cucurbitales, and Rosales, these four orders have been referred to as the nitrogen-fixing clade [13] (Figure 1). To account for the observation that nodulating lineages in this clade are interspersed among lineages that do not possess the symbiosis, two general hypotheses have been postulated [13]: (i) a single gain of nodulation in an ancestor of the nitrogen-fixing clade followed by massively parallel loss of this trait in most descendants, or (ii) parallel evolution of nodulation in some descendants and fewer losses. The first hypothesis has been almost universally dismissed, whereas the latter is widely accepted on the basis of two main arguments [14-24]. First, it comprises scenarios that require fewer evolutionary events and that are supported by phylogenetic ancestral state reconstruction studies [14-16,20,22]. Second, there is considerable variation among nodulating lineages in the type of microsymbiont, nodule ontogeny, and physiology [25-28]. For example, legume nodules are all infected by rhizobia and share a unique 'stem-like' ontogeny with a peripheral vascular system and a large central zone of infected cells. In contrast, nodules of the non-legume Parasponia are infected with the same rhizobial strains but have a 'lateral root-like' ontogeny with a central vascular bundle and infected cells in the periphery, similar to actinorhizal nodules [23]. Furthermore, distinct strategies evolved to provide low oxygen pressure to protect nitrogenase from oxidation. For example, most actinorhizal plants rely on mechanisms provided by the Frankia microsymbiont. Frankia spp. can differentiate into rigid infection structures, known as vesicles, that have a physical oxygen barrier, produce hopanoid-derivatives forming protective laminar lipid layers, and/or express truncated hemoglobin $\mathrm{HbO}$ that affect oxygen homeostasis $[25,29,30]$. In contrast, legumes, Parasponia, and the actinorhizal plant Casuarina (Fagales, Casuarinaceae, Casuarina glauca) evolved a plant-encoded mechanism to control oxygen homeostasis. This mechanism relies on adaptation of either class I (Parasponia) or class II hemoglobin genes (legumes and Casuarina) to function as oxygen transporters in infected nodule cells [26-28]. Taken together, these and other differences are often regarded as evidence that not all nodules are homologous and that nodulation therefore arose independently in different lineages [14,15,21,23,24,31].

Despite the general acceptance of the parallel evolution hypothesis, there are several issues that challenge this view. First, as parallel evolution can in principle occur in any plant, it does not explain why all nodulating species are confined to the nitrogen-fixing clade. To resolve this apparent conflict, it is commonly assumed that a genetic predisposition event leading to a precursor state for nodulation evolved in a common ancestor of the nitrogen-fixing clade around 110 million years ago (Mya). This precursor state facilitated the parallel evolution of nodulation in different descendant lineages [13,22]. Despite decades of research, this hypothetical precursor state for nodulation has remained elusive and lacks empirical support $[14,16,19,22]$. In our opinion, this renders the supposed predisposition a problematic explanation.

\section{Glossary}

Actinorhizal plants: plants that make nodules hosting a symbiosis with Frankia bacteria.

Diazotroph: a microorganism that is able to grow without external sources of fixed nitrogen by using nitrogenase.

Frankia: a genus of nitrogen-fixing, Gram-positive filamentous actinomycete bacteria that can engage a symbiosis with actinorhizal plants.

LCOs: lipo-chitooligosaccharides that act as symbiotic signals, that is, as Myc factors when produced by endomycorrhizal fungi or as Nod factors produced by rhizobia and Frankia.

NFP/NFR5: NOD FACTOR

PERCEPTION/NOD FACTOR

RECEPTOR 5 lysin motif (LysM) containing receptor kinase that is essential for Nod factor signaling in legumes.

NIN: NODULE INCEPTION transcription factor that is essential for nodule formation in legumes. Nitrogenase: bacterial enzyme complex responsible for catalyzing the reduction of nitrogen $\left(\mathrm{N}_{2}\right)$ to ammonia $\left(\mathrm{NH}_{3}\right)$.

\section{Nitrogen-fixing clade:} monophyletic group comprising the orders Fabales, Fagales, Cucurbitales, and Rosales and including all plant lineages with nitrogen-fixing root nodules.

Nod factors: symbiotic signaling LCOs produced by rhizobia and some Frankia bacteria that have nodulation genes.

Rhizobia: a polyphyletic group of diazotrophic Gram-negative rod-like $\alpha$ - and $\beta$-proteobacteria that can engage a symbiosis with nodulating legumes (Fabales) and Parasponia (Rosales, Cannabaceae). RPG: RHIZOBIUM-DIRECTED POLAR GROWTH, a long coiled-coil protein that is essential for rhizobial infection in legumes. 


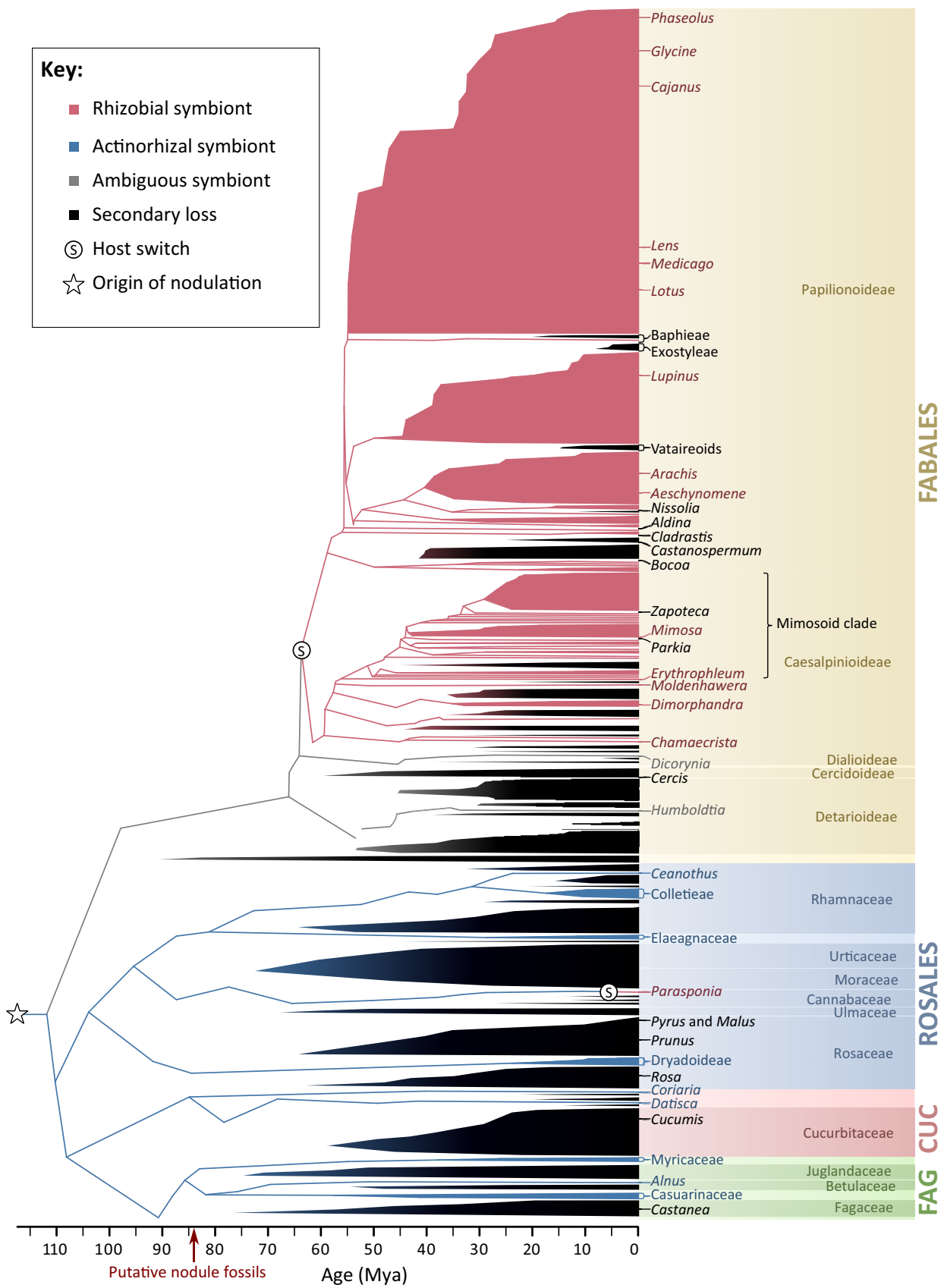

Trends in Plant Science

Figure 1. Single Gain and Massive Loss Hypothesis. Representation of the phylogenetic distribution of nodulating and non-nodulating plants in the nitrogen-fixing clade under the single gain and massive loss hypothesis. Phylogenetic tree of the nitrogen-fixing clade based on $[15,66,67]$; distribution of nodulating and non-nodulating plants based on [63]. Branch colors indicate putative host status and type of microsymbiont; color gradients indicate uncertainty in the phylogenetic placement of secondary losses. The legumes Dicorynia (Dialioideae) and Humboldtia (Detarioideae) have been reported to nodulate and may therefore represent additional 'relict' nodulators $[68,69]$. The putative host switch depicted in an ancestral legume is a minimum age estimate as it may have occurred as early as in an ancestor of all Fabales; the putative host switch depicted in an ancestral Parasponia is based on genetic changes supporting adaptations in hemoglobin required for oxygen transport in rhizobium symbiosis [11,27]. CUC, Cucurbitales; FAG, Fagales; Mya, million years ago. 
Second, scenarios with many parallel origins optimized on phylogenetic trees are in conflict with developmental and structural data [14]. For example, within legumes as many as four independent origins are reconstructed, even though all legumes share the same stem-like ontogeny, which is consistent with nodule homology $[18,19,22,23]$. Similarly, given their $\sim 85$-millionyear divergence, the two nodulating lineages in Cucurbitales Coriaria (Coriariaceae) and Datisca (Datiscaceae) are consistently reconstructed to represent independent origins, even though their nodule anatomy is very similar $[14,15,22,23]$. Also, the distinctive root-hair infection process shared by several species representing all three nodulating Fagales lineages suggests that their common ancestor had a similar characteristic [14,23,32]. Consequently, despite the evident phenotypic divergence between the main nodulating lineages that separated more than 100 Mya, developmental and structural data suggest far fewer and much older origins than those predicted based on phylogenetic considerations.

Third, phylogenetic ancestral state reconstructions generally imply a priori equal weight for gains and losses. However, considering the complexity of nitrogen-fixing nodules, it has often been acknowledged that an evolutionary gain of this trait is genetically much more difficult than a loss $[24,31,33]$. For example, single inactivating mutations in NFP/NFR5, NIN, and RPG each result in loss of functional nodules $[10,34,35]$. Thus, it would be more realistic to incorporate higher rates of losses versus gains when modeling the evolution of nodulation.

\section{A Single Gain of Nodulation}

Based on our current understanding of the phylogeny of the nitrogen-fixing clade, the hypothesis of a single gain of nodulation requires at least 7 losses in Fagales, 5 in Cucurbitales, 17 in Rosales, and 36 in Fabales (Figure 1). Assuming a single common nodulating ancestor readily explains why all nodulating species occur in one clade and, consequently, eliminates the need to hypothesize the highly speculative predisposition for nodulation, replacing it with the evolution of nodulation itself. Clearly, accurate reconstruction of evolutionary events that occurred $\sim 110$ Mya is difficult. Nevertheless, there are two main lines of evidence suggesting that the origin of nodulation is much older than generally assumed.

The first line of evidence comes from structural and developmental similarities across nodules from distantly related plants. Parasponia and some legume species host rhizobia in fixation threads, which show strong resemblance with infection structures found in actinorhizal nodules $[36,37]$. Fixation threads have been considered 'primitive', suggesting a common ancestral state [36]. Furthermore, the ontogeny of all nodules from Cucurbitales, Fagales, and Rosales species is very similar $[23,24,28]$. Given that these three orders form a clade (Figure 1), we believe that these similarities may be more readily explained by homology than by parallel or convergent similarity. The latter requires that developmental constraints favoring the evolution of a certain type of nodules arose in the common ancestor of this clade. This echoes the predisposition for nodulation in the entire nitrogen-fixing clade required by multiple gain scenarios, and for which there is no evidence. We therefore argue that these similarities have a stronger phylogenetic signal than what may be expected based on parallel evolution.

The second line of evidence can be found in the commonalities in symbiotic gene function between diverse nodulating lineages. Assuming that all nodules arose from a single gain, it can be predicted that the genes recruited for nodulation are orthologous [24]. For example, expression of the NIN transcription factor is essential to induce nodule organogenesis in legumes as well as in the actinorhizal plant Casuarina $[9,35,38]$. Moreover, Casuarina NIN can functionally complement a legume nin knockout mutant [9]. A recent study comparing genes with nodule-enhanced expression from medicago and Parasponia revealed a set of 290 
putatively orthologous genes that are used in both species [11]. These include key genes that are specifically required for nodulation such as NIN and RPG. Similarly, comparative transcriptomic studies revealed extensive commonalities in nodule-expressed genes between legumes and the actinorhizal plants Casuarina and Alnus (Fagales), although orthology was not assessed rigorously for these genes [17]. Such commonalities have been interpreted as cases of 'deep homology' or as leads to the supposed predisposition [17-19,39]. We rather advocate the simpler interpretation that these commonalities are the result of a single recruitment of all of the components of a homologous nodulation symbiosis [14,24]. In our opinion, these structural, molecular, and genetic data together suggest that the various nodulation phenotypes have a single origin.

The fossil record would provide clear evidence for the single gain hypothesis, if definitive fossilized roots bearing nodules were found that pre-dated ancestors in which independent origins of nodulation are hypothesized under the parallel origins hypothesis. Several fossilized root structures that strongly resemble multi-lobed nodules were dated at 84 Mya [40]. Given that nodule fossils are rare/absent even from more recent sediments in diverse and widespread lineages such as legumes, this date should be considered as a minimum bound for the age of nodulation if the fossils are truly nodules. Notably, it is much older than the crown age of any nodulating lineages (Figure 1) [15,22,41]. For example, legumes are the oldest and most diverse nodulating lineage, and abundant in the fossil record; but the earliest fossils that can be definitively assigned to the legume family appeared in the late Paleocene ( 65 Mya) [42]. Because nitrogen-fixing symbiotic nodules can be very difficult to distinguish from other root structures such as nematode root galls and ectomycorrhizal nodules [43], more and better fossil evidence are needed to confirm an early origin of nodulation (see Outstanding Questions).

\section{Parallel Loss of Symbiosis Genes in Non-nodulating Species}

Given the phylogenetic framework, a single gain hypothesis implies a massively parallel loss of the nodulation trait within the nitrogen-fixing clade $[13,18,24,33]$. It was previously hypothesized that the genomes of non-nodulating taxa could harbor 'fossil' evidence of nodulation, such as pseudogenization of genes functioning only in nodulation similarly as was found for loss of arbuscular mycorrhizal symbiosis $[19,44]$. Two recent phylogenomic studies found exactly that $[11,12]$. The first study compared the genome of Parasponia with its closest nonnodulating relative, Trema, and revealed that three genes that are essential for establishing nitrogen-fixing root nodules in legumes and actinorhizal plants, namely, NFP/NFR5, RPG, and NIN, are lost or pseudogenized in the Trema genome as well as in those of relatively distantly related non-nodulating Rosales species [11]. The second study compared genomes of nodulating and non-nodulating plants across the nitrogen-fixing clade and revealed a similar pattern of gene loss for NIN and RPG [12]. Orthologs of these genes occur outside the nitrogen-fixing clade where they must have non-symbiotic functions that remain unknown. Nevertheless, given our current understanding, within the nitrogen-fixing clade these genes are exclusively associated with nodulation, strongly suggesting that loss of these genes resulted from the loss of the nodulation trait. Consequently, absence of nodulation in these sampled species across the nitrogen-fixing clade likely represents a secondary loss rather than an ancestral state.

\section{Loss of Nodulation and Decreasing Global Atmospheric $\mathrm{CO}_{2}$}

Nodulation is an example of phenotypic plasticity in plants [45], turned on and off in nodulating species depending on the benefit provided by symbiotic bacteria relative to the cost of their maintenance. Although the relationship between phenotypic plasticity and adaptive evolution is 


\section{Trends in Plant Science}

complex [46], it seems reasonable to predict that, should abiotic and biotic conditions consistently disadvantage symbiosis in any nodulating plant species over a long period, the ability to nodulate would be lost. This is because, when nodulation is turned off, genes dedicated solely to the symbiosis, thus freed from purifying selection, would accumulate

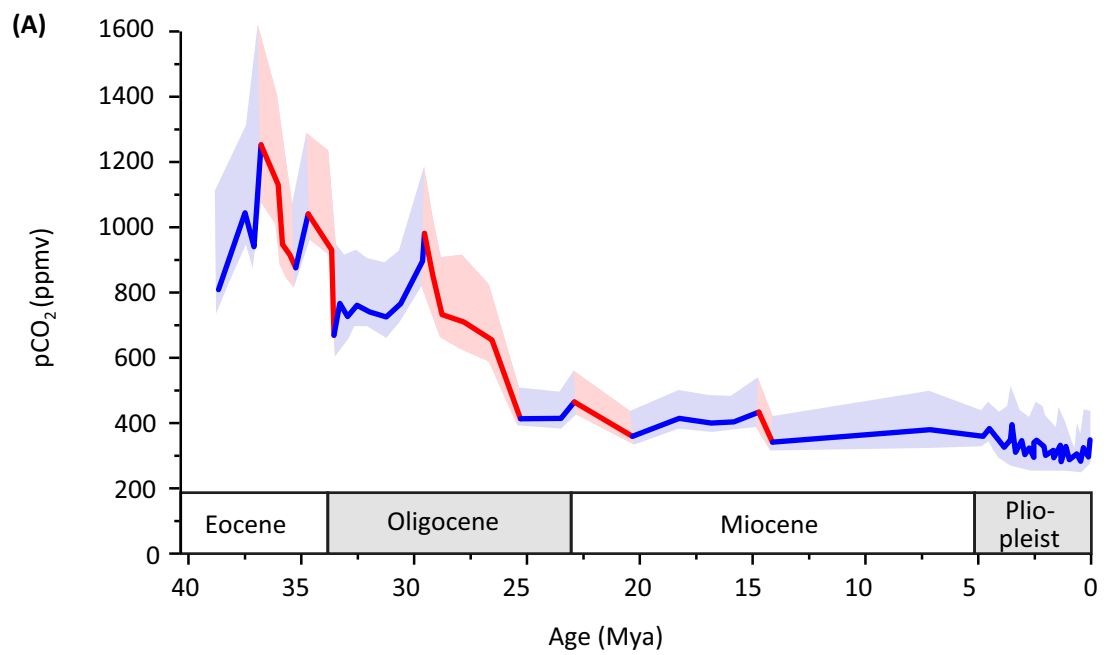

(B)
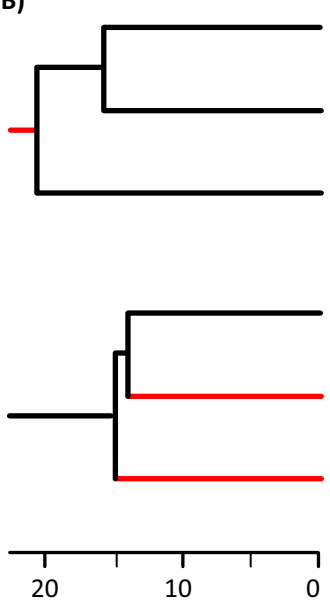

Age (Mya)

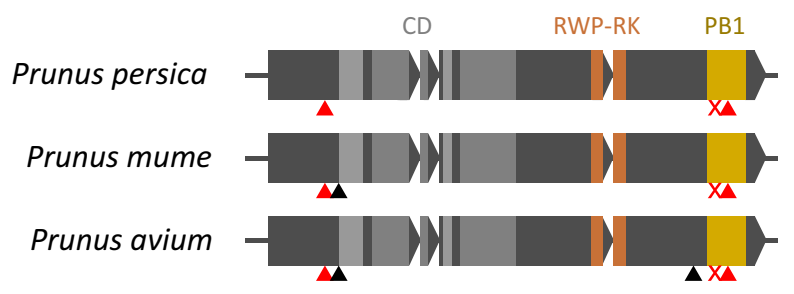

Parasponia andersonii

Trema levigata

Trema orientalis

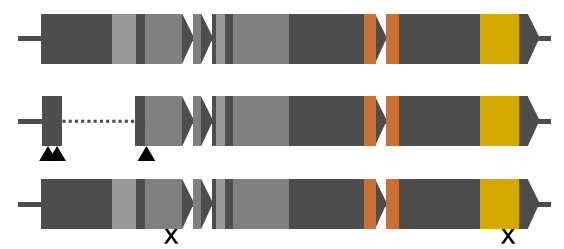

Trends in Plant Science

Figure 2. Decrease of Atmospheric $\mathrm{CO}_{2}$ and Pseudogenization of NIN in Non-nodulating Prunus and Trema Species. (A) Global atmospheric $\mathrm{CO}_{2}$ levels decreased over a period of 40 million years [56]. Significant drops are marked red and are most prominent in the Eocene-Oligocene transition 34 million years ago (Mya), through Late Oligocene 2923 Mya, and the Middle Miocene 14 Mya. Shaded areas indicate confidence intervals based on [56]. pCO2, partial pressure of carbon dioxide in parts per million volume; Plio-Pleist, Plio-Pleistocene. (B) Preliminary data on timing of parallel loss events based on mutations in the NIN gene. Shared loss-of-function mutations (marked in red) in NIN from Prunus species indicate that the associated loss event occurred before their divergence 21 Mya [70-73]. Independent loss-offunction mutations in NIN from two Trema species indicate that the associated loss events occurred after their divergence 17 Mya $[11,15]$. Phylogenetic branches along which putative loss events may have occurred are in red; NIN exon structure given in arrows (introns not in scale). CD, four conserved domains in gray; RWP-RK, conserved amino acid domain in orange; PB1, Phox and Bem1 domain in yellow. Triangles indicate frameshift mutations; $X$ 's indicate nonsense mutations resulting in premature in-frame stop codons. 
inactivating mutations through neutral processes and become pseudogenes. The major challenge for postulating many independent losses is therefore to identify a common factor that would cause nodulation, once an asset to plant growth, to become a liability in numerous unrelated nodulating plant lineages. Increased availability of fixed nitrogen, or development of 'cheating' bacteria that enter root nodules, but do not deliver nitrogen, have been postulated as possible factors driving loss of nodulation [12]. However, such scenarios would most probably act only locally, whereas widespread parallel loss is explained best by a factor acting globally and at geological timescales. One such factor is atmospheric $\mathrm{CO}_{2}$, which is the basis for photosynthesis. In contrast to other primary growth factors such as light, water, and nutrient availability, $\mathrm{CO}_{2}$ is relatively evenly distributed throughout the Earth's atmosphere, rendering it an inescapable selective force [47]. Changes in atmospheric $\mathrm{CO}_{2}$ levels have been postulated to drive the evolution of plant anatomy and physiology, such as C4 photosynthesis [48-51], stomatal density [52,53], and chemical defense [54,55]. Global atmospheric $\mathrm{CO}_{2}$ levels have generally been decreasing in the last 100 million years, since the time when nodulation is hypothesized to have arisen under the single origin scenario. During this general decrease, there have been periods of particularly steep decline during the Eocene-Oligocene transition 〜34 Mya, the Late Oligocene 29-23 Mya, and the Middle Miocene climate transition 14 Mya (Figure 2A) [56]. Consequently, given the high photosynthetic demands of symbiotic nitrogen fixation $[1,5]$ and the variability in photosynthetic efficiency of different species, $\mathrm{CO}_{2}$ could have become a limiting factor for plant growth for different lineages during these climatic transitions [51,53]. Decreasing $\mathrm{CO}_{2}$ would account not only for the loss of nodulation in diverse lineages but also for differences in the timing of loss, from very recent (e.g., Trema) to more ancient (e.g., Prunus) (Figure 2B).

\section{Concluding Remarks and Future Perspectives}

Based on the single gain and massive loss hypothesis, we predict loss of nodulation-specific genes in most non-nodulating species in the nitrogen-fixing clade. Testing this prediction requires a more comprehensive genome-scale analysis of additional non-nodulating lineages from the nitrogen-fixing clade as well as from related orders. Nevertheless, it is already clear that the patterns of maintenance and loss of nodulation genes are not consistent. For example, the non-nodulating plant jujube (Ziziphus jujuba) retains NIN [11,57], whereas the nodulating legume Arachis ipaensis lost RPG [12,58]. This suggests that nodulation genes that are maintained in non-nodulating species encode unknown non-symbiotic functions, whereas such genes can become dispensable in some nodulating lineages. In-depth understanding of the molecular functioning of these genes is required to obtain insights in such deviant evolutionary trajectories.

Assuming a single gain of nodulation, at least two switches between Frankia and rhizobial microsymbiotic partners must have occurred. We hypothesize that nodulation first evolved with an LCO-producing Frankia species rather than rhizobia, since Frankia has the intrinsic characteristics to protect nitrogenase from oxidation [25]. Subsequently, different proteobacteria obtained LCO biosynthesis genes by horizontal gene transfer [59-61], allowing them to compete with Frankia and independently infect ancestors of legumes and of Parasponia (Figure 1), where Burkholderia spp. ( $\beta$-proteobacteria) are considered to be more ancient microsymbionts than Rhizobiales ( $\alpha$-proteobacteria) [62]. Microsymbiont switches between Frankia and rhizobia almost certainly required genetic adaptations of the putative host plant. One such adaptation is the recruitment of hemoglobin genes to control oxygen homeostasis in the nodule, as rhizobia lack the oxygen-regulating features of Frankia [11,27]. It can be anticipated that several more adaptations, for example, in resistance responses, may have been essential.

\section{Outstanding Questions}

When, and how frequent, did parallel loss of nodulation occur?

Can nodulation be restored in nonnodulating plants by reintroducing lost symbiosis genes?

What was the ancestral nodule microsymbiont and in which lineages did host switches occur?

Can fossil evidence be found to substantiate: (i) occurrence of the nodulation trait >100 Mya, and (ii) subsequent massively parallel loss of this trait?

Under a massive loss scenario, what factor(s) determined that the currently symbiotic lineages retained the nodulation trait?

What are the functions of symbiosis genes such as NIN and RPG in nonnodulating species both inside and outside the nitrogen-fixing clade? 
Trends in Plant Science

Under a single gain and massively parallel loss scenario, the origin of nitrogen-fixing root nodulation should be further re-evaluated. Given massively parallel loss, the origin may have been even earlier than the nitrogen-fixing clade. Some indirect evidence supporting this hypothesis can be found in related orders within the fabid clade. First, there are unconfirmed studies on nodulating Zygophylloideae species (Zygophyllales, Zygophyllaceae) [63]. Second, stable isotope measurements of fossil wood from the Oligocene (23-34 Mya) suggested nitrogen fixation in Magnistipula (Malpighiales, Chrysobalanaceae) [50]. Experimental, phylogenomic, and fossil evidence is essential to assess whether these cases represent(ed) cases of nitrogen-fixing nodulation.

Based on our assumption that loss of nodulation genes is directly related to loss of nodulation, we predict that functional ancestral variants of these nodulation genes confer symbiotic potential to plants in the nitrogen-fixing clade. This can be tested by re-introducing such genes in non-nodulating plants and measuring symbiotic responses. Given their putatively recent loss of nodulation genes, close relationship with nodulating Parasponia [11], and availability of transformation protocols [64,65], Trema species are ideal candidates for such a re-engineering approach.

\section{Acknowledgements}

Erik Koenen kindly provided a preliminary time-calibrated phylogenetic tree for legumes that was incorporated in Figure 1. This work was supported by NWO-VICl (865.13.001) to R.G.

\section{References}

1. Hoffman, B.M. et al. (2014) Mechanism of nitrogen fixation by nitrogenase: the next stage. Chem. Rev. 114, 4041-4062

2. Nishida, H. and Suzaki, T. (2018) Nitrate-mediated control of root nodule symbiosis. Curr. Opin. Plant Biol. 44, 129-136

3. Ferguson, B.J. et al. (2018) Legume nodulation: the host controls the party. Plant Cell Environ. Published online May 28, 2018. http://dx.doi.org/10.1111/pce.13348

4. McCoy, J.M. et al. (2018) Nitrogen fertilization of soybean affects root growth and nodulation on two soil types in Mississippi. Commun. Soil Sci. Plant Anal. 49, 181-187

5. Taylor, B.N. and Menge, D.N.L. (2018) Light regulates tropical symbiotic nitrogen fixation more strongly than soil nitrogen. Nat. Plants 4, 655-661

6. Downie, J.A. (2014) Legume nodulation. Curr. Biol. 24, R184-R190

7. Coba de la Peña, T. et al. (2017) The symbiosome: legume and rhizobia co-evolution toward a nitrogen-fixing organelle? Front. Plant Sci. 8, 2229 http://dx.doi.org/10.3389/fpls.2017.02229

8. Vernié, T. et al. (2015) The NIN transcription factor coordinates diverse nodulation programs in different tissues of the Medicago truncatula root. Plant Cell 27, 3410-3424

9. Clavijo, F. et al. (2015) The Casuarina NIN gene is transcriptionally activated throughout Frankia root infection as well as in response to bacterial diffusible signals. New Phytol. 208, 887-903

10. Arrighi, J.-F. et al. (2008) The RPG gene of Medicago truncatula controls Rhizobium-directed polar growth during infection. Proc. Natl. Acad. Sci. U. S. A. 105, 9817-9822

11. van Velzen, R. et al. (2018) Comparative genomics of the nonlegume Parasponia reveals insights into evolution of nitrogen-fixing rhizobium symbioses. Proc. Natl. Acad. Sci. U. S. A. 115, E4700-E4709

12. Griesmann, M. et al. (2018) Phylogenomics reveals multiple losses of nitrogen-fixing root nodule symbiosis. Science Published online May 24, 2018. http://dx.doi.org/10.1126/science. aat1743

13. Soltis, D.E. et al. (1995) Chloroplast gene sequence data suggest a single origin of the predisposition for symbiotic nitrogen fixation in angiosperms. Proc. Natl. Acad. Sci. U. S. A. 92, $2647-2651$
14. Swensen, S.M. (1996) The evolution of actinorhizal symbioses: evidence for multiple origins of the symbiotic association. Am. J. Bot. 83, 1503-1512

15. Li, H.-L. et al. (2015) Large-scale phylogenetic analyses reveal multiple gains of actinorhizal nitrogen-fixing symbioses in angiosperms associated with climate change. Sci. Rep. 5, 14023-14023

16. Roy, A. and Bousquet, J. (1996) The evolution of the actinorhizal symbiosis through phylogenetic analysis of host plants. Acta Bot. Gallica 143, 635-650

17. Hocher, V. et al. (2011) Transcriptomics of actinorhizal symbioses reveals homologs of the whole common symbiotic signaling cascade. Plant Physiol. 156, 700-711

18. Doyle, J.J. (2011) Phylogenetic perspectives on the origins of nodulation. Mol. Plant Microbe Interact. 24, 1289-1295

19. Doyle, J.J. (2016) Chasing unicorns: nodulation origins and the paradox of novelty. Am. J. Bot. 103, 1-4

20. Jeong, S.C. et al. (1999) Molecular phylogenies of plants and Frankia support multiple origins of actinorhizal symbioses. Mol Phylogenet. Evol. 13, 493-503

21. Doyle, J.J. (1998) Phylogenetic perspectives on nodulation: evolving views of plants and symbiotic bacteria. Trends Plant Sci. 3, 473-478

22. Werner, G.D.A. et al. (2014) A single evolutionary innovation drives the deep evolution of symbiotic $\mathrm{N}_{2}$-fixation in angiosperms. Nat. Commun. 5, 4087-4087

23. Svistoonoff, S. et al. (2014) Actinorhizal root nodule symbioses: what is signalling telling on the origins of nodulation? Curr. Opin. Plant Biol. 20, 11-18

24. Doyle, J.J. (1994) Phylogeny of the legume family: an approach to understanding the origins of nodulation. Annu. Rev. Ecol. Syst. 25, 325-349

25. Silvester, W.B. et al. (2008) Oxygen responses, hemoglobin, and the structure and function of vesicles. In Nitrogen-Fixing Actinorhizal Symbioses (Pawlowski, K. and Newton, W.E., eds), pp. 105-146, Springer Netherlands

26. Ott, T. et al. (2005) Symbiotic leghemoglobins are crucial for nitrogen fixation in legume root nodules but not for general plant growth and development. Curr. Biol. 15, 531-535 
Trends in Plant Science

27. Sturms, R. et al. (2010) Trema and Parasponia hemoglobins reveal convergent evolution of oxygen transport in plants. Biochemistry 49, 4085-4093

28. Pawlowski, K. and Demchenko, K.N. (2012) The diversity of actinorhizal symbiosis. Protoplasma 249, 967-979

29. Huss-Danell, K. (1997) Tansley review no. 93. Actinorhizal symbioses and their $\mathrm{N}_{2}$ fixation. New Phytol. 136, 375-405

30. Coats, V. et al. (2009) Truncated hemoglobins in Frankia Ccl3: effects of nitrogen source, oxygen concentration, and nitric oxide. Can. J. Microbiol. 55, 867-873

31. Swensen, S.M. and Mullin, B.C. (1997) Phylogenetic relationships among actinorhizal plants. The impact of molecular systematics and implications for the evolution of actinorhizal symbioses. Physiol. Plant 99, 565-573

32. Santi, C. et al. (2013) Biological nitrogen fixation in non-legume plants. Ann. Bot. 111, 743-767

33. Swensen, S.M. and Benson, D.R. (2008) Nitrogen-fixing actinorhizal symbioses. In Evolution of Actinorhizal Host Plants and Frankia Endosymbionts (Pawlowski, K. and Newton, W.E., eds), pp. 73-104, Springer

34. Madsen, E.B. et al. (2003) A receptor kinase gene of the LysM type is involved in legume perception of rhizobial signals. Nature $425,637-640$

35. Schauser, L. et al. (1999) A plant regulator controlling development of symbiotic root nodules. Nature 402, 191-195

36. Sprent, J.I. et al. (2013) From North to South: a latitudinal look at legume nodulation processes. S. Afr. J. Bot. 89, 31-41

37. Behm, J.E. et al. (2014) Parasponia: a novel system for studying mutualism stability. Trends Plant Sci. 19, 757-763

38. Marsh, J.F. et al. (2007) Medicago truncatula NIN is essential for rhizobial-independent nodule organogenesis induced by autoactive calcium/calmodulin-dependent protein kinase. Plant Physiol. 144, 324-335

39. Soyano, T. and Hayashi, M. (2014) Transcriptional networks leading to symbiotic nodule organogenesis. Curr. Opin. Plant Biol. 20, 146-154

40. Herendeen, P.S. et al. (1999) A preliminary conspectus of the Allon flora from the late Cretaceous (late Santonian) of central Georgia, U.S.A. Ann. Mo. Bot. Gard. 86, 407-471

41. Lavin, M. et al. (2005) Evolutionary rates analysis of Leguminosae implicates a rapid diversification of lineages during the tertiary. Syst. Biol. 54, 575-594

42. Bruneau, A. et al. (2008) Phylogenetic patterns and diversification in the caesalpinioid legumes. Botany 86, 697-718

43. Sprent, J.I. (2001) Nodulation in Legumes, Royal Botanic Gardens, Kew

44. Delaux, P.M. et al. (2014) Comparative phylogenomics uncovers the impact of symbiotic associations on host genome evolution. PLoS Genet. 10, e1004487

45. Goh, C.-H. et al. (2013) The impact of beneficial plant-associated microbes on plant phenotypic plasticity. J. Chem. Ecol. 39, 826-839

46. Price, T.D. et al. (2003) The role of phenotypic plasticity in driving genetic evolution. Proc. Biol. Sci. 270, 1433-1440

47. Gerhart, L.M. and Ward, J.K. (2010) Plant responses to low $\left[\mathrm{CO}_{2}\right]$ of the past. New Phytol. 188, 674-695

48. Sage, R.F. et al. (2012) Photorespiration and the evolution of C4 photosynthesis. Annu. Rev. Plant Biol. 63, 19-47

49. Heckmann, D. (2016) C4 photosynthesis evolution: the conditional Mt. Fuji. Curr. Opin. Plant Biol. 31, 149-154

50. Gulbranson, E.L. et al. (2017) Nitrogen-fixing symbiosis inferred from stable isotope analysis of fossil tree rings from the Oligocene of Ethiopia. Geology 45, 687-690

51. Christin, P.-A. et al. (2008) Oligocene $\mathrm{CO}_{2}$ decline promoted $\mathrm{C} 4$ photosynthesis in grasses. Curr. Biol. 18, 37-43
52. Steinthorsdottir, M. et al. (2016) Fossil plant stomata indicate decreasing atmospheric $\mathrm{CO}_{2}$ prior to the Eocene-Oligocene boundary. Clim. Past 12, 439-454

53. Kürschner, W.M. et al. (2008) The impact of Miocene atmospheric carbon dioxide fluctuations on climate and the evolution of terrestrial ecosystems. Proc. Natl. Acad. Sci. U. S. A. 105, 449-453

54. Noctor, G. and Mhamdi, A. (2017) Climate change, $\mathrm{CO}_{2}$, and defense: the metabolic, redox, and signaling perspectives. Trends Plant Sci. 22, 857-870

55. Johnson, S.N. and Züst, T. (2018) Climate change and insect pests: resistance is not futile? Trends Plant Sci. 23, 367-369

56. Zhang, Y.G. et al. (2013) A 40-million-year history of atmospheric $\mathrm{CO}_{2}$. Philos. Trans. A Math. Phys. Eng. Sci. 371, 20130096

57. Huang, J. et al. (2016) The Jujube genome provides insights into genome evolution and the domestication of sweetness/acidity taste in fruit trees. PLoS Genet. 12, e1006433

58. Bertioli, D.J. et al. (2016) The genome sequences of Arachis duranensis and Arachis ipaensis, the diploid ancestors of cultivated peanut. Nat. Genet. 48, 438-446

59. Persson, T. et al. (2015) Candidatus Frankia Datiscae Dg1, the actinobacterial microsymbiont of Datisca glomerata, expresses the canonical nod genes nodABC in symbiosis with its host plant. PLoS One 10, e0127630

60. Garrido-Oter, R. et al. (2018) Modular traits of the Rhizobiales roo microbiota and their evolutionary relationship with symbiotic rhizobia. Cell Host Microbe 24, 155-167.e5

61. Remigi, P. et al. (2016) Symbiosis within symbiosis: evolving nitrogen-fixing legume symbionts. Trends Microbiol. 24, 63-75

62. Bontemps, C. et al. (2010) Burkholderia species are ancient symbionts of legumes. Mol. Ecol. 19, 44-52

63. Tedersoo, L. et al. (2018) Global database of plants with rootsymbiotic nitrogen fixation: NodDB. J. Veg. Sci. 29, 560-568

64. Cao, Q. et al. (2012) Efficiency of Agrobacterium rhizogenesmediated root transformation of Parasponia and Trema is temperature dependent. Plant Growth Regul. 68, 459-465

65. van Zeiil, A. et al. (2018) CRISPR/Cas9-mediated mutagenesis of four putative symbiosis genes of the tropical tree Parasponia andersonii reveals novel phenotypes. Front. Plant Sci. 9, 284

66. The Legume Phylogeny Working Group (2017) A new subfamily classification of the Leguminosae based on a taxonomically comprehensive phylogeny. Taxon 66, 44-77

67. Larson-Johnson, K. (2016) Phylogenetic investigation of the com plex evolutionary history of dispersal mode and diversification rates across living and fossil Fagales. New Phytol. 209, 418-435

68. Moreira, F.M.D.S. et al. (1992) Occurrence of nodulation in legume species in the Amazon region of Brazil. New Phytol. $121,563-570$

69. Lalani Wijesundara, T.I. et al. (2000) Rhizobiology and nitrogen fixation of some tree legumes native to Sri Lanka. Biol. Fertil. Soils 30, 535-543

70. Xiang, Y. et al. (2017) Evolution of Rosaceae fruit types based on nuclear phylogeny in the context of geological times and genome duplication. Mol. Biol. Evol. 34, 262-281

71. Zhang, Q. et al. (2012) The genome of Prunus mume. Nat. Commun. 3, 1318

72. Shirasawa, K. et al. (2017) The genome sequence of sweet cherry (Prunus avium) for use in genomics-assisted breeding. DNA Res. 24, 499-508

73. Verde, I. et al. (2013) The high-quality draft genome of peach (Prunus persica) identifies unique patterns of genetic diversity, domestication and genome evolution. Nat. Genet. 45, 487-494 